\title{
Drell-Yan differential cross section measurement at CMS
}

\section{Kyeongpil Lee*}

Seoul National University (KR)

E-mail: kplee@cern.ch

\begin{abstract}
Measurement of the differential Drell-Yan cross sections in the dimuon channel is presented. It is based on proton-proton collision data at $13 \mathrm{TeV}$ recorded with the CMS detector at the LHC, and the integrated luminosity of the data is $2.8 \mathrm{fb}^{-1}$. The differential cross section in the dilepton mass range from 15 to $3000 \mathrm{GeV}$ is measured and corrected to the full phase space and the detector acceptance. These measurements are compared to higher order perturbative QCD predictions and show good agreement with the predictions.
\end{abstract}

38th International Conference on High Energy Physics 3-10 August 2016

Chicago, USA

${ }^{*}$ Speaker. 


\section{Introduction}

The Drell-Yan (DY) process is the process in which lepton pairs are produced via $\mathrm{Z} / \gamma^{*}$ exchange. The differential cross section of this process is theoretically well established up to nextto-next-to-leading order (NNLO) in quantum chromodynamics (QCD). Precisely measured cross sections can not only be an effective input for the constraint on the parton distribution functions (PDFs) but also be used in various LHC new physics search [1] as a major background process. The differential cross sections of DY process were previously measured in ATLAS [2,3] and CMS Collaborations $[4,5,6]$ at $\sqrt{s}=7$ and $\sqrt{s}=8 \mathrm{TeV}$. This analysis presents the differential cross section $d \sigma / d m$ of DY process in dimuon channel from $15 \mathrm{GeV}$ to $3000 \mathrm{GeV}$ with total 43 bins using proton-proton collision data collected with the CMS detector at $\sqrt{s}=13 \mathrm{TeV}$. The integrated luminosity of the data is about $2.8 \mathrm{fb}^{-1}$. The cross section per each mass bin is calculated using the formula $\sigma=N /\left(A \cdot \varepsilon \cdot \mathscr{L}_{\text {int }}\right)$ where $N$ means the number of signal yields, and $A, \varepsilon$ and $\mathscr{L}_{\text {int }}$ are the acceptance, efficiency and the integrated luminosity of the data respectively. Various Monte-Carlo (MC) samples are used in this analysis in order to estimate the signal and backgrounds. Signal MC sample is generated by aMC@NLO generator, and considered backgrounds are QCD, W+Jets, $t \bar{t}$, $t W, \bar{t} W, Z / \gamma^{*} \rightarrow \tau \tau$ and diboson processes. The order of analysis procedure is the event selection, background estimation, corrections, estimation of systematic uncertainties and results.

\section{Event Selection and Background Estimation}

The purpose of event selection is to select two oppositely charged and isolated muons originated from DY process. The selection criteria is similar to the one used in Run1 analysis. The events passing single isolated muon trigger with larger than $20 \mathrm{GeV}$ transverse momentum $\left(P_{T}\right)$ are selected. For the muons, leading and subleading muons should have $P_{T}>22$ and $10 \mathrm{GeV}$ respectively with pseudorapidity $|\eta|<2.4$. CMS standard track quality conditions and relative tracker isolation are used for the muon selection [7].

Dominant background is top quark events for entire mass range. For the other backgrounds, QCD and W+Jets events come into the signal region mainly in low mass region, and small contributions from diboson and $Z / \gamma^{*} \rightarrow \tau \tau$ exist in $Z$ peak region. These backgrounds are estimated using a control data sample except for $\mathrm{WZ}$ and $\mathrm{ZZ}$. The backgrounds from top quark and electroweak processes are estimated by e $\mu$ method described in [9], and QCD and W+Jets events are estimated by misidentification rate method [8]. WZ and ZZ events are based on MC prediction and they are normalized to the integrated luminosity of the data. The left plot in Fig. 1 is the dimuon mass distribution after event selection. Generally data and the signal and background predictions show good agreement in all mass region.

\section{Corrections and Systematic Uncertainties}

Unfolding correction is firstly applied after the background subtraction from the data. The purpose of this correction is to correct the bin migration among invariant mass bins due to the detector resolution effect. Response matrix is calculated using signal MC sample. D'Agostini's iteration method [11] is used for the unfolding correction. 

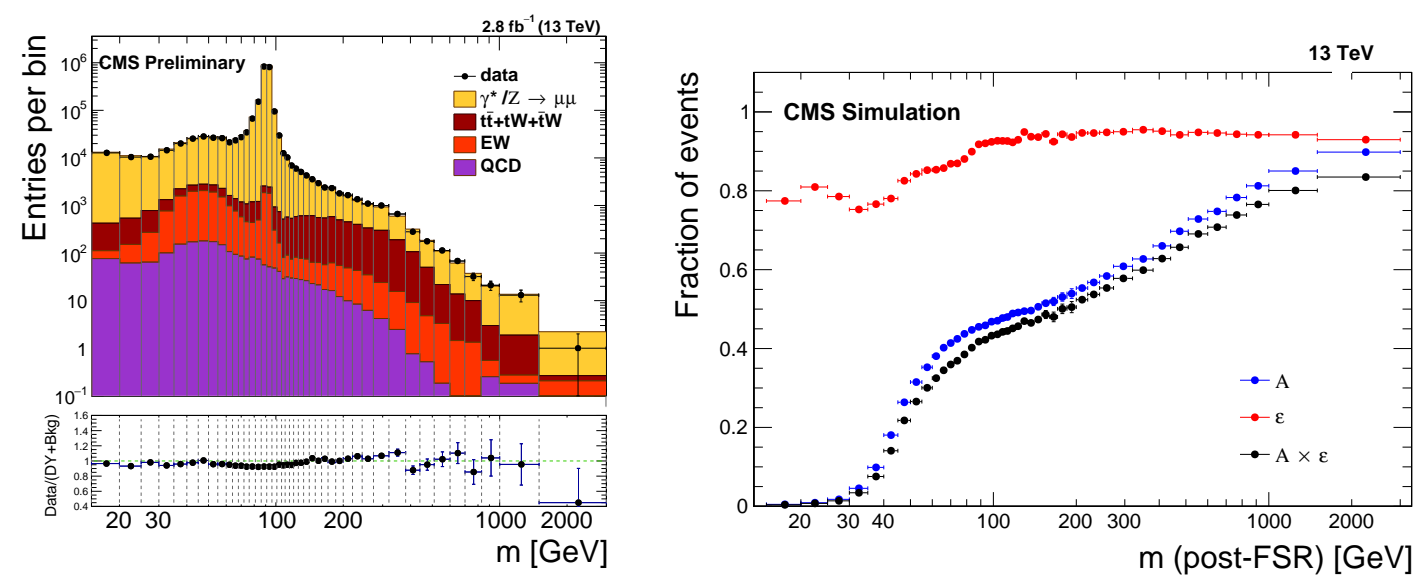

Figure 1: Left: Dimuon invariant mass distribution including all estimated backgrounds after event selection. Right: Acceptance and efficiency per dimuon invariant mass bin.

Next step is acceptance and efficiency corrections. The right plot in Fig. 1 shows the acceptance and efficiency per each mass bin. Acceptance goes up to $90 \%$ in the highest mass bin, and efficiency is generally from 80 to $90 \%$. In order to take into account the difference of the efficiency between data and MC, efficiency scale factors determined by Tag and Probe method are also applied on the data [10].

Lastly, another unfolding correction is applied on the distribution to account for the final state radiation (FSR) effect. Momentum of photons near a muon within $\Delta R=0.1$ are added to the muon's momentum, which is so called "dressed lepton". Response matrix is calculated using signal MC sample whose FSR effect is simulated by Pythia8, and matrix inversion method is used for FSR unfolding.

In the low mass region, systematic uncertainty from efficiency scale factor is dominant, and the size of the uncertainty is about 3\%. In the $\mathrm{Z}$ peak region, FSR correction is the dominant systematic uncertainty, but still it is smaller than the luminosity uncertainty. Statistical uncertainty becomes dominant in high mass region because of lack of statistics. Fig. 3 in Ref. [9] shows the details about the uncertainties.

\section{Results and Conclusion}

Fig. 2 is the differential cross section from 15 to $3000 \mathrm{GeV}$. Left plot is the results in full phase space with FSR correction, and it is compared to the aMC@NLO and NNLO prediction calculated by FEWZ with NNPDF3.0. Right one is the results within the detector acceptance to minimize the theoretical dependence of the experimental result, compared to the aMC@NLO result. Generally, theoretical prediction and experimental result show good agreement within the uncertainties. More detail information is available in Ref. [9].

\section{Acknowledgement}

K. P. Lee is supported in part by the National Research Foundation of Korea (NRF) funded by 

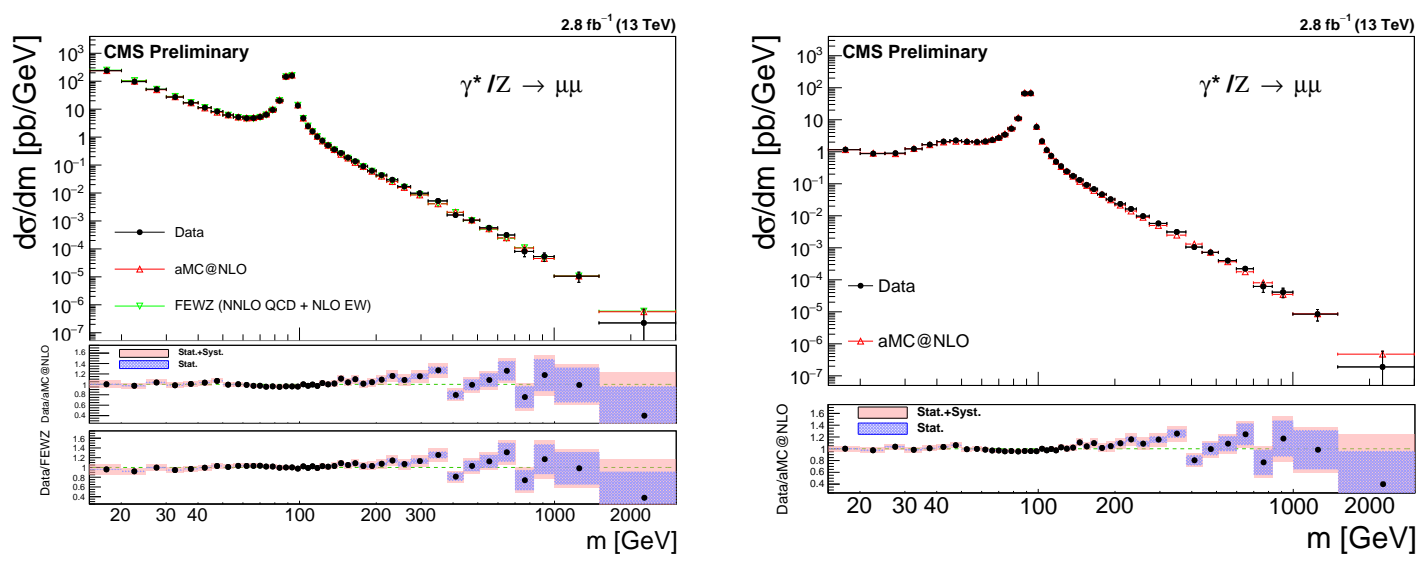

Figure 2: Comparison of the differential cross sections between experimental result and theoretical prediction. Left plot is the result after applying all corrections. Right one is the result within the detector acceptance. In the ratio plot, violet and red boxes denote statistical uncertainty only and full uncertainty including systematic uncertainty.

the Korea government (NRF-2015R1C1A1A01053087 and NRF-2015R1A4A1042542).

\section{References}

[1] CMS Collaboration, Search for heavy narrow dilepton resonances in pp collisions at $\sqrt{s}=7 \mathrm{TeV}$ and $\sqrt{s}=8 \mathrm{TeV}$, Phys. Lett. B 720 (2013) 63

[2] ATLAS Collaboration, Measurement of the high-mass Drell-Yan differential cross-section in pp collisions at $\sqrt{s}=7 \mathrm{TeV}$ with the ATLAS detector, Phys. Lett. B 725 (2013) 223

[3] ATLAS Collaboration, Measurement of the low-mass Drell-Yan differential cross section at $\sqrt{s}=7$ TeV using the ATLAS detector, JHEP 06 (2014) 112

[4] CMS Collaboration, Measurement of the Drell-Yan Cross Section in pp Collisions at $\sqrt{s}=7 \mathrm{TeV}$, JHEP 10 (2011) 007

[5] CMS Collaboration, Measurement of the differential and double-differential Drell-Yan cross sections in proton-proton collisions at $\sqrt{s}=7 \mathrm{TeV}$, JHEP 12 (2013) 030

[6] CMS Collaboration, Measurement of differential and double-differential Drell-Yan cross sections in proton-proton collisions at $\sqrt{s}=8 \mathrm{TeV}$, Eur. Phys. J. C 75 (2015) 147

[7] CMS Collaboration, Performance of CMS muon reconstruction in pp collision events at $\sqrt{s}=7 \mathrm{TeV}$, JINST 7 (2012) P10002

[8] CMS Collaboration, Search for physics beyond the standard model in dilepton mass spectra in proton-proton collisions at $\sqrt{s}=8 \mathrm{TeV}$, JHEP 04 (2015) 025

[9] CMS Collaboration, Measurement of the differential Drell-Yan cross section in proton-proton collisions at $\sqrt{s}=13 \mathrm{TeV}$, CMS Physics Analysis Summary CMS-PAS-SMP-16-009, 2016

[10] CMS Collaboration, Measurement of the inclusive $W$ and $Z$ production cross sections in pp collisions at $\sqrt{s}=7 \mathrm{TeV}$ with the CMS detector, JHEP 10 (2011) 132

[11] G. D'Agostini, A multidimensional unfolding method based on Bayes' theorem, Nucl. Instrum. Meth. A 362 (1995) 487 